\title{
Categorização dos casos de violência contra crianças em um município piauiense
}

\author{
Categorization of cases of violence against children in a city in the state of Piaui, Brazil
}

Samaria de Sousa Nolêto ${ }^{1}$ (D), Mohema Duarte de Oliveira ${ }^{1}$ (D), Francisca Rayla Teles Meneses ${ }^{1}$ (D) Ivaldo da Silva Muniz ${ }^{1}$ (D),

Margarida Maria de Sousa Macedo ${ }^{1}$ (๑) Shirley Ribeiro de Assis ${ }^{1}(\mathbb{D}$

\section{RESUMO}

Objetivo: Identificar os tipos de violência contra crianças notificados em um município piauiense, no ano de 2017. Métodos: Trata-se de uma pesquisa do tipo exploratória, descritiva, de natureza aplicada e abordagem quantitativa, desenvolvida no hospital regional do município de Floriano, Piauí. A amostra do estudo foi composta de 181 fichas de notificação compulsória de violência infantil registradas no ano de 2017. Os dados foram coletados por meio de um roteiro baseado na ficha de notificação, desenvolvida de setembro de 2017 a julho de 2018. Resultados: O tipo de violência prevalente foi a negligência $(80,66 \%)$, sendo a recorrência ausente em $87,3 \%$. Foram registrados $6,63 \%$ de casos de violência sexual isolada ou associada à outra forma, como a física e a psicológica; desse total, houve predomínio do assédio sexual (25\%). Conclusões: A percepção da dimensão da violência infantil ainda é insuficiente, pois os dados evidenciam apenas uma pequena parcela da realidade. Os resultados da pesquisa possibilitaram o direcionamento e a referência para a elaboração de ações que objetivaram enfrentar a violência contra crianças por meio do conhecimento do perfil das vítimas atingidas.

Palavras-chave: maus-tratos infantis; notificação de abuso; atitude do pessoal de saúde; percepção; monitoramento epidemiológico.

\begin{abstract}
Objective: To identify the types of violence against children reported in a city in the state of Piauí in 2017. Methods: This is an exploratory, descriptive, and quantitative approach of applied nature developed at the regional hospital of the municipality of Floriano, Piauí, Brazil. The sample consisted of 181 compulsory notification reports of child violence registered in 2017. Data were collected through a script based on the notification form being developed from September 2017 to July 2018. Results: The prevalent type of violence was negligence (80.66\%), and recurrence was absent in $87.3 \%$. There were $6.63 \%$ of cases of isolated or associated sexual violence, such as physical and psychological, of which sexual harassment predominated (25\%). Conclusions: The true perception of the dimension of child violence is still insufficient, since data are often only a small part of reality. The results of the research made possible the direction and reference for the elaboration of actions that aimed to confront violence against children by knowing the profile of the affected victims. Keywords: child abuse; mandatory reporting; attitude of health personnel; perception; epidemiological monitoring.
\end{abstract}

\section{INTRODUÇÃO}

A violência pode ocorrer quando uma das partes envolvidas na situação assume o papel de superior, ou seja, quando determinadas diferenças são transformadas em possíveis desigualdades, estabelecendo uma condição de dominação e submissão. Considera-se que, em determinadas situações de violência, o ser humano dominado é considerado como um objeto. ${ }^{1}$

Qualquer situação que seja caracterizada pela omissão de atos realizados por pais ou parentes que resulte em transtornos psicológicos, físicos ou sexuais é considerada violência contra crianças e adolescentes. A condição de vulnerabilidade

${ }^{1}$ Universidade Estadual do Piauí, Centro de Ciências da Saúde - Floriano (PI), Brasil.

Autor correspondente: Samaria de Sousa Nolêto - Universidade Estadual do Piauí, Centro de Ciências da Saúde - BR-343, s/n. CEP: 64800-000 - Campo Velho - Floriano (PI), Brasil - E-mail: samarinha_noleto@ hotmail.com

Recebido em 16/08/2017 - Aceito para publicação em 26/02/2019. 
a qual crianças e adolescentes estão predispostos resulta da convivência com adultos que as violentam. Nessa situação inexiste o direito de oportunidade, a liberdade, o respeito, a dignidade ou a chance de um crescimento saudável., ${ }^{2,3}$

A violência contra crianças e adolescentes envolve maus-tratos, negligência, abandono, abuso sexual, exploração comercial e trabalho infantil. No Brasil, esses casos aumentam a cada dia, porém ganharam a maior visibilidade apenas nos últimos anos. A criação e a implantação do Estatuto da Criança e do Adolescente (ECA) tornaram possível que situações como essas sejam vistas como violação dos direitos humanos. ${ }^{4}$

De acordo com Moreira et al., ${ }^{5}$ um espaço propício para a ocorrência de agressões é o ambiente familiar, pela possibilidade de se desenvolver relações desiguais de poder. As estratégias utilizadas para o enfrentamento da violência requerem um manejo minucioso, pois grande parte das agressões ocorre no contexto familiar e possui elevado grau de reincidência, configurando-se como um acontecimento que perdura por muito tempo e que gera grandes prejuízos.

As principais agressões que ocorrem contra crianças envolvem injúrias faciais resultantes de maus-tratos, com graves repercussões físicas e psicológicas. Entre as possíveis lesões, destacam-se as queimaduras geradas por utensílios domésticos ou alimentos quentes, as contusões, as lesões orofaciais, as lacerações e o desvio da abertura bucal. A presença de lesões como escaras nos cantos da boca e equimoses nas bochechas sugere, respectivamente, amordaçamento e agressões por socos ou tapas. ${ }^{6,7}$

Portanto, o objetivo desta pesquisa foi identificar os tipos de violência contra crianças notificados em um município piauiense, no ano de 2017.

\section{MÉTODOS}

Trata-se de um estudo do tipo exploratório, descritivo, de natureza aplicada e abordagem quantitativa, desenvolvido no município de Floriano, Piauí, a partir das fichas de notificação de violência contra crianças armazenadas no setor epidemiológico de um hospital público.

O período de coleta dos dados foi de abril a junho de 2018, referentes às informações das notificações realizadas de janeiro a dezembro de 2017.

A população da pesquisa foi formada pelos 181 casos de notificação por violência contra crianças registrados na instituição hospitalar no ano de 2017. Admitiram-se erro amostral de 5\% e nível de confiança de $95 \%$ caso surgissem perdas durante a aplicação dos critérios de inclusão e de exclusão, no intuito de garantir a confiabilidade do estudo.

Nesta investigação, adotou-se como critério de inclusão os registros de notificação compulsória de violência contra crianças (menores de 12 anos) durante o ano de 2017, devidamente preenchidos e datados. Concomitantemente, considerou-se como critério de exclusão as fichas de notificação de menores de 12 anos que, mesmo preenchidas, estivessem rasuradas ou inelegíveis, impossibilitando a coleta de dados.
A saber, mesmo após utilização dos critérios de inclusão e de exclusão, todas as fichas de notificação continham informações suficientes para atender aos objetivos da pesquisa, não havendo perda amostral. Assim, fizeram parte do estudo todos os casos de notificação contra crianças no ano de 2017.

As informações obtidas após a coleta dos dados foram submetidas à análise descritiva a partir do objetivo da presente investigação. Todos os dados foram traduzidos em números para melhor estudo e demonstração.

O projeto foi pautado na Resolução ${ }^{\circ}$ 466/12 e na Resolução $\mathrm{n}^{\circ}$ 510/16 do Conselho Nacional de Saúde (CNS), que dispõem sobre as diretrizes e as normas regulamentadoras de pesquisas que envolvam seres humanos, obedecendo aos princípios da Bioética. Após a autorização pelo Núcleo de Educação Permanente do hospital, a pesquisa foi submetida à análise e ao julgamento do Comitê de Ética em Pesquisa da Universidade Estadual do Piauí (CEP-UESPI), sendo aprovado com CAAE no 84985318.0.0000.5209.

\section{RESULTADOS}

No que concerne aos tipos de violência notificados, foi possível observar grande associação entre os diversos tipos de violência. Na presente investigação, de acordo com o Gráfico 1, a violência mais prevalente foi a negligência $(80,88 \%)$, seguida de violência física/negligência $(11,05 \%)$, sexual $(3,87 \%)$ e sexual/psicológica $(2,21 \%)$. As violências física, física/psicológica, física/sexual, bem como a psicológica/doméstica, tiveram mesma proporção, representando $0,55 \%$ dos casos notificados.

Como pode ser observado na Tabela 1 , foram registrados $12(6,63 \%)$ casos de violência sexual isolada ou associada à outra forma, como a física e a psicológica; desse total, houve predomínio do assédio sexual (25\%). O estupro e a bolinação acometeram $16,66 \%$ das vítimas; já o ato libidinoso e o assédio sexual/pornografia infantil tiveram a mesma proporção $(8,34 \%)$ entre as notificações de violência contra crianças no ano de 2017. Entre as vítimas desse tipo de violência, houve predomínio do sexo feminino $(83,33 \%)$.

Quanto à recorrência da violência, foi observado que apenas 3,86\% dos casos notificados no ano de 2017 ocorreram mais de uma vez. A maioria dos casos teve essa variável como ignorada $(87,3 \%)$.

Considerada um problema de saúde pública, a violência infantil é um agravo universal que acomete grande número de indivíduos e resulta em malefícios e danos irreparáveis para inúmeras vítimas, além de se apresentar de forma variada e silenciosa.

\section{DISCUSSÃO}

Um fator de elevada importância e que está associado com a ocorrência de violência infantil é a utilização de álcool e outras drogas. De acordo com Oliveira, ${ }^{8}$ estudos demonstram a recorrência de agressões contra crianças relacionadas com drogadição. O uso de álcool associado a outras drogas leva a 
comportamentos violentos que resultam na opressão daqueles que possuem menor poder, como as mulheres e as crianças.

Corroborando com a investigação realizada por Rates et al., ${ }^{7}$ referente a uma análise das notificações compulsórias de violência infantil, evidenciou-se a negligência como a forma de violência mais notificada. Em seguida, foi possível observar casos de violência física, sexual e psicológica. O sexo masculino apresenta maior vulnerabilidade para ser acometido por negligência e violência física, enquanto o sexo feminino é mais atingido pela violência sexual e psicológica.

Estudo realizado na Unidade de Emergência Referenciada Pediátrica do Hospital de Clínicas da Universidade Estadual de Campinas (Unicamp) evidenciou a negligência como a forma mais frequente de maus-tratos entre crianças, envolvendo cerca de $65 \%$ dos casos. ${ }^{9}$

A negligência se configura como uma das formas de violência mais notificadas em diversas investigações, e abrange toda e qualquer categoria de omissão dos pais ou responsáveis de suprir as necessidades básicas e indispensáveis para o pleno desenvolvimento social, físico e emocional das crianças. ${ }^{10}$

Para Acioli et al., ${ }^{11}$ a elevada notificação de casos de negligência e violência física pode ser justificada pela maior facilidade na detecção desses tipos de maus-tratos, pois eles deixam indícios que demonstram a ausência de cuidados gerais com o menor e marcas visíveis de violência, características ausentes em outros tipos.

Diversos fatores podem contribuir para a invisibilidade do fenômeno de violência, dificultando sua identificação e notificação. Entre esses fatores, podem-se citar o silêncio intrafamiliar, a presença ou a ausência de lesões visíveis, além da sensibilidade de profissionais que atuam na área da saúde ou da educação. ${ }^{12}$

É importante destacar que os profissionais que atuam na área da saúde devem se atentar para qualquer suspeita de violência infantil, preparando-se para lidar e identificar indicadores de maus-tratos mais complicados, além de sinais não tão evidentes, como a violência física e a negligência.

Segundo Apostólico et al., ${ }^{13}$ a violência sexual é uma modalidade de agressão que predomina entre as meninas, sendo cometida principalmente no contexto privado do lar, envolvendo medo, vergonha e relações de subordinação.

As vítimas da violência sexual são mais propícias ao acometimento por outros tipos de violência, bem como pelas lesões físicas sofridas. Além disso, essas vítimas são mais propensas a contrair alguma infecção sexualmente transmissível, HIV ou, ainda, engravidar, resultado de abuso sexual. A grande magnitude alcançada por essa temática tem gerado grandes desafios para a sociedade atual. ${ }^{14}$

Apesar de as notificações de violência psicológica serem reduzidas, elas merecem destaque por serem um tipo de maus-tratos de difícil percepção e detecção, tanto por parte das

Tabela 1. Caracterização da violência sexual contra crianças notificada em um hospital público no ano de 2017, Floriano, Piauí, 2018 (n=12).

$\begin{array}{lcc}\text { Variável } & \text { Frequência } & \% \\ \text { Tipo de violência sexual } & & \\ \text { Assédio sexual } & 3 & 25 \\ \text { Estupro } & 2 & 16,66 \\ \text { Bolinação } & 2 & 16,66 \\ \text { Ato libidinoso } & 2 & 16,66 \\ \text { Assédio sexual/pornografia infantil } & 1 & 8,34 \\ \text { Assédio sexual/sexo oral } & 1 & 8,34 \\ \text { Ignorado } & 1 & 8,34\end{array}$

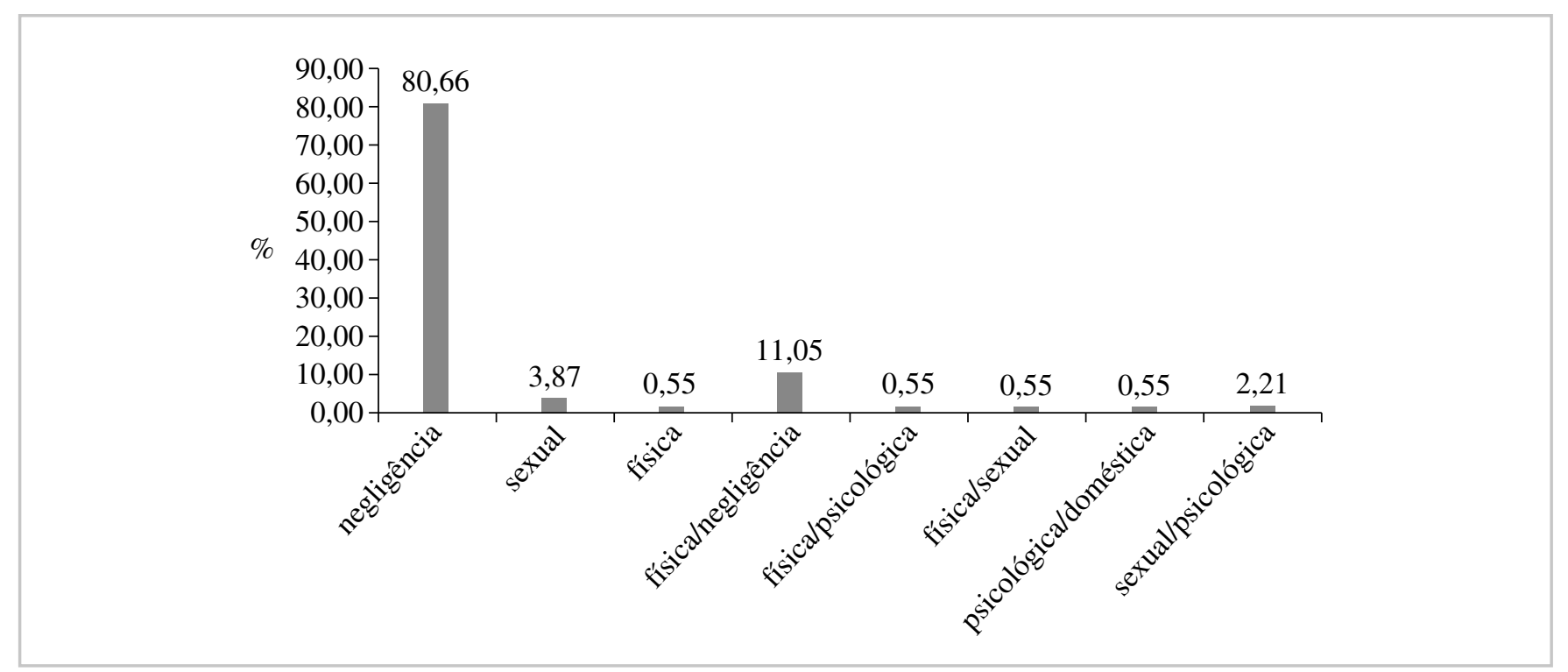

Gráfico 1. Categorização da violência contra crianças notificada em um hospital público no ano de 2017 (n=181), Floriano, Piauí, 2018. 
próprias vítimas, que, muitas vezes, não percebem que estão passando por essa situação, quanto das pessoas que convivem com as vítimas, que poderiam contribuir com a denúncia. ${ }^{15}$

Ressalta-se que, embora a violência psicológica seja pouco mencionada na literatura, ela demanda muita atenção por parte dos profissionais, já que seus sinais são pouco evidentes e não constitui a principal categoria para denúncia, mesmo quase sempre acompanhada por outros tipos de violência. A identificação de casos suspeitos ou confirmados deve acontecer o mais breve possível, pois as consequências dessa ocorrência são gravíssimas para a saúde mental da vítima, sendo necessária maior caracterização desse tipo de vitimização contra as crianças. ${ }^{16}$

Apesar da proporção de recorrência encontrada ser pequena, não deve ser ignorada, pois a taxa de reincidência constitui um importante indicativo de enfrentamento e combate à violência infantil, uma vez que possibilita a identificação da repetitividade desses agravos e permite a aquisição de subsídios para a elaboração e o planejamento de atividades e ações que evitem atos sucessivos de maus-tratos, já que a violência não se restringe apenas a uma ocorrência. ${ }^{17}$

De acordo com estudo desenvolvido por Sommer et al. ${ }^{18}$ referente à caracterização da violência contra crianças e adolescentes, 35,7\% das ocorrências foram a primeira notificação e 33,9\% registraram a recorrência da violência. Segundo Oliveira ${ }^{8}$ em investigação realizada na cidade de Recife, Pernambuco, 33\% das crianças e dos adolescentes sofreram maus-tratos mais de dez vezes, e $20 \%$ eram menores de 10 anos na primeira notificação.

Na infância, a convivência em um ambiente de violência, seja entre os familiares, seja com a própria criança, representa riscos significativos para a vida dos menores. Os modelos de conduta vivenciados pelas crianças podem ser reproduzidos por elas, passando a utilizá-los como forma natural de resolução de conflitos e considerar socialmente aceitáveis. ${ }^{13}$

\section{CONCLUSÕES}

A violência infantil constitui um grave problema de saúde pública que demanda enfoque da sociedade para que todas as crianças possam usufruir e viver a própria infância de forma absoluta e plena, possibilitando seu crescimento e desenvolvimento saudável. Diante disso, surge a necessidade da percepção precoce dos casos de violência por parte da comunidade e também dos profissionais de saúde, identificando, conduzindo e notificando as ocorrências.

A violência infantil pode ser caracterizada como um tipo de agravo que contraria e desrespeita todos os direitos fundamentais das crianças. Muitas vezes considerado um ato de disciplina, a violência contra crianças é um problema de cunho estadual, familiar e social.

A característica repetitiva da violência retrata que medidas interventivas nos tipos de ocorrência não têm resultado satisfatório, já que o contexto da violência infantil abrange as relações familiares. Sendo assim, existe maior urgência no desenvolvimento de medidas protetivas, educação e pre- venção destinadas a famílias e crianças que convivam com situações de violência.

A percepção da dimensão da violência contra crianças ainda é insuficiente, pois os dados evidenciam apenas uma pequena parcela da realidade. As notificações de maus-tratos ainda ocorrem de forma desestruturada. Muitos casos não chegam aos serviços de saúde e, ainda, podem ser mantidos como fato sigiloso, principalmente quando o responsável pela agressão é uma pessoa próxima da vítima. Todos esses fatos contribuem para a dificuldade em se conhecer a real magnitude desses agravos.

Os resultados da presente pesquisa possibilitaram o direcionamento e a referência para a elaboração de metas e ações que objetivam enfrentar a violência infantil por meio do conhecimento do perfil das crianças atingidas. Além disso, fornece informações sobre a realidade dessa problemática para os profissionais de saúde, reforçando a necessidade e a indispensabilidade da elaboração do plano de cuidados, sensibilizando-os nos momentos de recepção de um caso e de realização da notificação.

\section{REFERÊNCIAS}

1. Silva PA. Notificação da violência intrafamiliar contra crianças e adolescentes como exercício de poder e resistência [tese]. Rio Grande: Universidade Federal de Rio Grande; 2015.

2. Minayo MCS. O desafio do conhecimento. Pesquisa qualitativa em saúde. $14^{\mathrm{a}}$ ed. São Paulo: Hucitec; Rio de Janeiro: ABRASCO; 2012.

3. Pesce R. Violência familiar e comportamento agressivo e transgressor na infância: uma revisão da literatura. Ciênc Saúde Coletiva. 2009;14(2):507-18. doi: http://dx.doi. org/10.1590/S1413-81232009000200019

4. Monteiro EMLM, Brandão Neto W, Gomes IMB, Freitas RBN, Brady CL, Moraes MUB. Violência contra criança e adolescente: rompendo o silêncio. Rev Rene. 2009;10(3):107-16.

5. Moreira TNF, Martins CL, Feuerwerker LCM, Schraiber LB. A construção do cuidado: o atendimento às situações de violência doméstica por equipes de Saúde da Família. Saúde Soc. 2014;23(3):814-27. doi: http://dx.doi.org/10.1590/S0104-12902014000300007

6. Sousa GFP, Carvalho MMP, Granville-Garcia AF, Gomes MNC, Ferreira JMS. Conhecimento de acadêmicos em odontologia sobre maus-tratos infantis. Odonto. 2012;20(40):109-17. doi: http://dx.doi. org/10.15603/2176-1000/odonto.v20n40p109-117

7. Rates SMM, Melo EM, Mascarenhas MDM, Malta DC. Violência infantil: uma análise das notificações compulsórias, Brasil 2011. Ciênc Saúde Coletiva. 2015;20(3):655-65. doi: http://dx.doi. org/10.1590/1413-81232015203.15242014

8. Oliveira RNG. Violência de gênero e necessidades em saúde: limites e possibilidades da estratégia saúde da família [tese]. São Paulo: Universidade de São Paulo; 2011. 
9. Zambon MP, Jacintho ACA, Medeiros MM, Guglielminetti R, Marmo DB. Violência doméstica contra crianças e adolescentes: um desafio. Rev Assoc Méd Bras. 2012;58(4):465-71. doi: http://dx.doi. org/10.1590/S0104-42302012000400018

10. Veloso LUP, Silva LCL, Sousa CR, Rodrigues PL. Perfil de violência em crianças de 0 a 9 anos atendidas em um hospital público. Rev Enferm UFPI. 2015;4(1):97-105. doi: https://doi.org/10.26694/reufpi.v4i1.3529

11. Acioli RML, Lima MLC, Braga MC, Pimentel FC, Castro AG. Violência intrafamiliar contra crianças e adolescentes: identificação, manejo e conhecimento da rede de referência por fonoaudiólogo em serviços públicos de saúde. Rev Bras Saúde Mater Infant. 2011;11(1):21-8. doi: http://dx.doi.org/10.1590/S151938292011000100003

12. Oliveira HC, Pinto Junior EP, Tavares LT, Guimarães MAP, Oliveira MND. Notificação compulsória de violência sexual contra crianças e adolescentes. Arq Ciênc Saúde. 2015;22(4):26-30. doi: https://doi. org/10.17696/2318-3691.22.4.2015.59

13. Apostólico MR, Nóbrega CR, Guedes RN, Fonseca RMGS, Egry EY. Características da violência contra a criança em uma capital brasileira. Rev Latino-Am Enfermagem. 2012;20(2):266-73.
14. Brasil. Ministério da Saúde. Linha de cuidado para a atenção integral à saúde de crianças, adolescentes e suas famílias em situação de violência. Orientações para gestores e profissionais de saúde. Versão preliminar. Brasília: Ministério da Saúde; 2010.

15. Garbin CAS, Queiroz APDG, Rovida TAS, Saliba O. A violência familiar sofrida na infância: uma investigação com adolescentes. Psicol Rev. 2012;18(1):107-18. doi: http://dx.doi.org/10.5752/P.1678-9563.2012v18n1p107

16. Garbin CAS, Rovida TAS, Joaquim RC, Paula AM, Queiroz APDG. Violência denunciada: ocorrências de maus tratos contra crianças e adolescentes registradas em uma unidade policial. Rev Bras Enferm. 2011;64(4):665-70. doi: http://dx.doi.org/10.1590/ S0034-71672011000400006

17. Martins CBG, Jorge MHPM. A violência contra crianças e adolescentes: características epidemiológicas dos casos notificados aos Conselhos Tutelares e programas de atendimento em município do Sul do Brasil, 2002 e 2006. Epidemiol Serv Saúde. 2009;18(4):315-34. doi: http://dx.doi.org/10.5123/S1679-49742009000400002

18. Sommer D, Franciscatto LG, Getelina CO, Salvador K. Caracterização da violência contra crianças e adolescentes: indicativos para a prática do enfermeiro. Rev Enferm. 2017;13(13):14-28. 